\title{
The influence of neuronal electrical activity on the mammalian central clock metabolome
}

\author{
M. Renate Buijink ${ }^{1} \cdot$ Michel van Weeghel $^{1,2} \cdot$ M. Can Gülersönmez ${ }^{2}$. Amy C. Harms ${ }^{2}$. Jos H. T. Rohling ${ }^{1}$. \\ Johanna H. Meijer ${ }^{1}$. Thomas Hankemeier ${ }^{2}$. Stephan Michel ${ }^{1}$
}

Received: 10 May 2018 / Accepted: 31 August 2018 / Published online: 17 September 2018

(c) The Author(s) 2018

\begin{abstract}
Introduction Most organisms display circadian rhythms in physiology and behaviour. In mammals, these rhythms are orchestrated by the suprachiasmatic nucleus (SCN). Recently, several metabolites have emerged as important regulators of circadian timekeeping. Metabolomics approaches have aided in identifying some key metabolites in circadian processes in peripheral tissue, but methods to routinely measure metabolites in small brain areas are currently lacking.

Objective The aim of the study was to establish a reliable method for metabolite quantifications in the central circadian clock and relate them to different states of neuronal excitability.

Methods We developed a method to collect and process small brain tissue samples $\left(0.2 \mathrm{~mm}^{3}\right)$, suitable for liquid chromatography-mass spectrometry. Metabolites were analysed in the SCN and one of its main hypothalamic targets, the paraventricular nucleus (PVN). Tissue samples were taken at peak (midday) and trough (midnight) of the endogenous rhythm in SCN electrical activity. Additionally, neuronal activity was altered pharmacologically.

Results We found a minor effect of day/night fluctuations in electrical activity or silencing activity during the day. In contrast, increasing electrical activity during the night significantly upregulated many metabolites in SCN and PVN.

Conclusion Our method has shown to produce reliable and physiologically relevant metabolite data from small brain samples. Inducing electrical activity at night mimics the effect of a light pulses in the $\mathrm{SCN}$, producing phase shifts of the circadian rhythm. The upregulation of metabolites could have a functional role in this process, since they are not solely products of physiological states, they are significant parts of cellular signalling pathways.
\end{abstract}

Keywords Circadian clock metabolites $\cdot$ Suprachiasmatic nucleus $\cdot$ Neuronal activity $\cdot$ Small brain samples · ZICcHILIC-MS

M. Renate Buijink and Michel van Weeghel as well as Thomas Hankemeier and Stephan Michel have contributed equally to this work as shared first and last authors respectively.

Electronic supplementary material The online version of this article (https://doi.org/10.1007/s11306-018-1423-z) contains supplementary material, which is available to authorized users.

Stephan Michel

s.michel@lumc.nl

1 Department of Cellular and Chemical Biology, Leiden University Medical Center, Einthovenweg 20, 2333 ZC Leiden, The Netherlands

2 Analytical BioSciences and Metabolomics, Division of Systems Biomedicine and Pharmacology, Leiden Academic Centre for Drug Research (LACDR), Leiden University, Einsteinweg 55, 2333 CC Leiden, The Netherlands

\section{Introduction}

Almost all organisms express circadian rhythms in physiology and behaviour defined by a period of about $24 \mathrm{~h}$. In mammals, these circadian rhythms are controlled by the suprachiasmatic nucleus (SCN), a small brain area located just above the optic chiasm (Moore and Eichler 1972; Stephan and Zucker 1972). The SCN is synchronized to the 24-h environmental cycle mainly by light. In the absence of external cues like light or temperature cycles, the neuronal network of the SCN sustains an autonomous circadian rhythm in electrical activity and neurotransmitter release (Gillette and Reppert 1987; Nishino et al. 1976). These signals from the SCN serve as a temporal reference for the rest of the body and orchestrate circadian rhythms throughout the brain and body, including sleep-wake cycles (Ibuka 
and Kawamura 1975; Moore 2007), food intake and energy metabolism (Coomans et al. 2013; Kalsbeek et al. 2011; Nagai et al. 1978). Disturbances in circadian rhythms have been found to be associated with many diseases, including metabolic syndrome (Rudic et al. 2004; Turek et al. 2005) and neurodegenerative diseases (Ju et al. 2013; Kondratova and Kondratov 2012; Musiek et al. 2015; Wulff et al. 2010).

Neurons in the SCN exhibit robust autonomous rhythmicity, which is controlled on several levels. There are known intracellular oscillators on the level of gene expression, cytosol and electrical activity. First, on the level of gene expression, the "molecular clock"-consisting of clock genes and proteins-constitutes a well-described core transcriptiontranslation feedback loop (TTFL), which has a cycle time of about $24 \mathrm{~h}$ (Sangoram et al. 1998). Second, in addition to the TTFL there are several cytosolic oscillators like $\mathrm{Ca}^{2+}$, cAMP and redox state (Ikeda et al. 2003; O'Neill et al. 2008; Wang et al. 2012). The redox oscillator is well-preserved among species and is considered to be a link between metabolic state, and both the molecular clock and membrane excitability (Wang et al. 2012). Lastly, an essential part of the SCN clockwork is the electrical activity of SCN neurons. Electrical activity modifies the molecular clock and vice versa and this interaction between membrane and clock genes is essential for sustained rhythm generation (Allen et al. 2017; Colwell 2011).

Neurons in the SCN are electrically active during the day, and relatively silent during the night in both diurnal and nocturnal animals (Inouye and Kawamura 1979; Sato and Kawamura 1984). One of the main targets for the SCN activity is the paraventricular nucleus (PVN) of the hypothalamus, which receives both paracrine as well as GABAergic and glutamatergic input from the SCN, and controls feeding behaviour and plasma glucose level (Kalsbeek et al. 2004; Santoso et al. 2017; Tousson and Meissl 2004). While the rhythm in electrical activity of extra-SCN areas in the hypothalamus has been found to be reversed in phase, the only study that directly measured electrical activity in the PVN has found the electrical activity rhythm to be in phase with the SCN (Inouye and Kawamura 1979; Kubota et al. 1981; Tousson and Meissl 2004).

Metabolites are products of cellular regulatory processes, and their relative levels are therefore indicative of the metabolic state of a cell. They are an attractive target to study since they are identical across species, and organisms differ less in their metabolome than in their genome or proteome. Several metabolites, such as cAMP (O'Neill et al. 2008), and metabolic pathways, such as the pentose phosphate pathway (Rey et al. 2016) are considered to be relevant for proper functioning of the circadian clockwork. Metabolomics has proven to be useful to study circadian clock function in liver and blood of mice (Eckel-Mahan et al. 2012; Minami et al. 2009), and has been applied to blood samples of humans to investigate the effect of sleep deprivation (Davies et al. 2014). However, until now there are no metabolomics studies of specific brain areas like the SCN, an important constraint being their small size, especially in commonly used animal models such as the mouse. Although there are complex techniques to measure metabolites with mass spectrometry in small samples, and even single cells (Lapainis et al. 2009; Qi et al. 2018), more generally applicable methods for small tissue samples are lacking. Therefore, we aimed to design a suitable method for tissue sampling and metabolite extraction for the analysis of metabolites with liquid chromatography-mass spectrometry (LC-MS) in small brain areas (Fig. 1). Using this method, we have studied the metabolic profiles of the $\mathrm{SCN}$, as well as the $\mathrm{PVN}-\mathrm{a}$ first relay area to control many physiological functions. We were interested in endogenous differences between day and night, as well as the effect of exogenously modulating neuronal activity on the metabolic profile of these brain areas.

Since we expected differences in energy metabolism, we focused our analytical approach on small polar metabolites, including metabolites from the TCA cycle, glycolysis, pentose phosphate and nucleotide pathways. For the metabolic profiling of the SCN and the PVN we used zwitterionic hydrophilic interaction liquid chromatography mass spectrometry (ZIC-cHILIC-MS), which is especially suitable for the analysis of anionic polar metabolites in complex aqueous matrices. In this study we show that it is feasible to measure metabolites in very small tissue samples $\left(0.2 \mathrm{~mm}^{3}\right)$. We could reliably measure and identify 35 metabolites in SCN and PVN tissue. We found only one metabolite (malate) significantly upregulated in the day compared to the night condition. Exposure to high extracellular $\mathrm{K}^{+}$at midnight upregulated many of the metabolites measured in SCN and PVN tissue, including all metabolites of the TCA cycle.

\section{Method}

\subsection{Animals and housing}

This study was performed in accordance with the Dutch law on animal welfare. The permit was issued by the animal experiments committee Leiden (DEC 12250). Male C57BL/6 mice were held in the animal facility of the Leiden University Medical Center, with food and water available ad libitum. Mice were kept in 24-h light-dark cycles with $12 \mathrm{~h}$ of light (50-100 lx; Osram truelight TL) and $12 \mathrm{~h}$ of darkness. Mice were approximately 3 months old (84-91 days) at the time of the experiments. 
Fig. 1 Overview of tissue sampling and processing. a For extraction and measurements of metabolites in SCN and PVN tissue, the brain was first isolated from the mouse, and the hypothalamic region, containing the SCN was cut in $250 \mu \mathrm{m}$ thick slices. These slices were incubated in ACSF, or ACSF with either $0.5 \mu \mathrm{M}$ TTX or $15 \mathrm{mM} \mathrm{K}^{+}$. b After incubation, the SCN and PVN were extracted from the slices by a sample corer. Because of the tight control over the thickness of the slices $(250 \mu \mathrm{m})$ and the diameter of the punch $(500 \mu \mathrm{m})$, the volume of the sample was constant $(0.2$ $\mathrm{mm}^{3}$ ). Punches were placed in 50/50 methanol/water and directly snap frozen in $\mathrm{N}_{2}$. Samples were kept at $-80 \mathrm{C}$ until metabolite extraction. c Metabolites were extracted from the tissue by using a liquid-liquid extraction method with $100 \mu \mathrm{L}$ chloroform added to the $100 \mu \mathrm{L} 50 / 50$ methanol/ water. Samples were homogenized in an ice-cold sonication bath for $3 \times 10 \mathrm{~min}$. Between sonication, samples were snap frozen for a short period in $\mathrm{N}_{2}$. Proteins were cleared from the solution by centrifuging. The top layer was transferred to a clean $0.5 \mathrm{~mL}$ tube and dried in a vacuum concentrator. $\mathbf{d}$ The dried samples were then reconstituted in $20 \mu \mathrm{L}$ of $60 / 40$ methanol/water and analysed by liquid chromatography through a ZIC-cHILIC column, followed by mass spectrometry
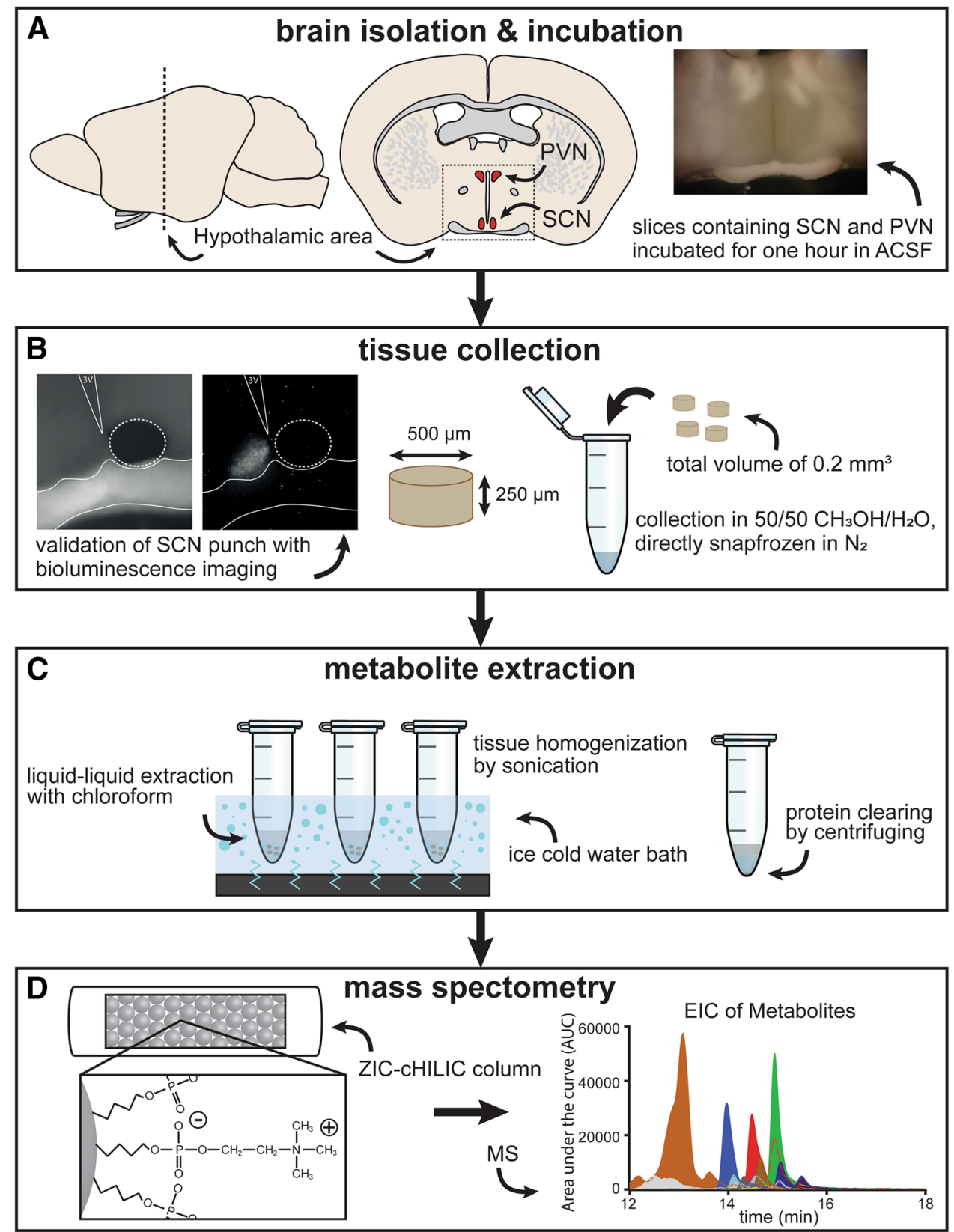

\subsection{SCN and PVN tissue sampling}

For the collection of SCN and PVN tissue, mice were sacrificed either an hour before midday (ZT6) or midnight (ZT18). The mice were fasted for $4 \mathrm{~h}$ before the start of the experiment. After decapitation, the brain was quickly dissected and placed in ice cold, low $\mathrm{Ca}^{2+}$ and high $\mathrm{Mg}^{2+}$ artificial cerebral spinal fluid (ACSF), containing (in $\mathrm{mM}$ ): $\mathrm{NaCl}$ (116.4), $\mathrm{KCl}$ (5.4), $\mathrm{NaH}_{2} \mathrm{PO}_{4}$ (1.0), $\mathrm{MgSO}_{4}(0.8), \mathrm{CaCl}_{2}$ (1.0), $\mathrm{MgCl}_{2}$ (4.0), $\mathrm{NaHCO}_{3}$ (23.8), D-glucose (15.1) and $5 \mathrm{mg} / \mathrm{L}$ gentamicin (Sigma Aldrich) saturated with $95 \% \mathrm{O}_{2}$ $-5 \% \mathrm{CO}_{2}$ (pH 7.4). From each individual animal, two consecutive coronal slices of $250 \mu \mathrm{m}$ were cut with a VT $1000 \mathrm{~S}$ vibratome (Leica). The brain slices were incubated for an hour in either normal ACSF containing $2 \mathrm{mM} \mathrm{CaCl}_{2}$ and no $\mathrm{MgCl}_{2}$ (ZT6; ZT18), or normal ACSF with TTX $(0.5 \mu \mathrm{M}$; ZT6) or higher levels of $\mathrm{K}^{+}(15 \mathrm{mM}$; ZT18), at room temperature ( $n=6$ for all groups). This method, including the incubation in ACSF is widely used as preparation for physiological experiments on SCN tissue (Nakamura et al. 2012; Michel et al. 2013). Slices were kept cold while collecting the SCN and PVN punches within a few minutes after incubation. We collected bilateral punches of $\varnothing 500 \mu \mathrm{m}$ (sample corer, 19-gauge, Fine Science Tools; adapted from Lee et al. 2010), from two consecutive slices, resulting in 4 punches of both the SCN and PVN, with a total volume of 
$0.2 \mathrm{~mm}^{3}$ from one animal per sample. This standardized and reproducible volume rendered weighing the samples unnecessary. Punches were directly placed in $100 \mu \mathrm{L}$ ice cold $50 / 50$ methanol $/ \mathrm{H}_{2} \mathrm{O}$ containing $5 \mu \mathrm{M}$ of internal standards (succinic acid-D4, 13C5-valine, 13C4-15N2-asparagine, 13C5-glutamine and 15N2-UMP), and snapfrozen in liquid nitrogen (adapted from Nemes et al. 2011). The combination of methanol and snapfreezing ensured adequate quenching of enzymatic activity, is suitable for metabolite extraction, and can be used for the ZIC-cHILIC column. Samples were stored at $-80^{\circ} \mathrm{C}$ until further processing. Figure $1 \mathrm{~b}$ shows a bright field and a bioluminescence image from a brain slice with one of the two SCN cores removed by punch.

\subsection{Metabolite extraction}

Metabolites were extracted using a combination of chemical and mechanical techniques (Fig. 1b, c). First, a liquid-liquid extraction (LLE) was performed by adding $100 \mu \mathrm{L}$ of chloroform to the samples in methanol-water. Samples were vortexed, then sonicated in an ice-cold sonication bath for $10 \mathrm{~min}$, followed by snapfreezing in liquid nitrogen. This procedure was repeated 3 times. The samples were then centrifuged for $10 \mathrm{~min}$ at $14.000 \mathrm{~g}$ at $4{ }^{\circ} \mathrm{C}$ to remove proteins from the solution. Before and after the sonication and centrifuging, samples were kept on ice. From the $100 \mu \mathrm{L}$ polar top layer $80 \mu \mathrm{L}$ was transferred to a $1.5 \mathrm{~mL}$ tube and dried in a vacuum concentrator (Labconco, MO, USA) for $3 \mathrm{~h}$. The samples were reconstituted in $20 \mu \mathrm{L}$ of methanol/ $\mathrm{H}_{2} \mathrm{O}$ solution $(60 / 40)$.

\subsection{Metabolite analysis}

Metabolites were analysed using liquid chromatography followed by mass spectrometry. For the analysis, an Agilent 1200 ultra-high-pressure liquid chromatography system (Agilent technologies, Santa Clara, CA, USA) coupled to a SCIEX TripleTOF 5600 quadrupole-time-of-flight mass spectrometer (Framingham, MA, USA) was used. Samples were kept at $10{ }^{\circ} \mathrm{C}$ in the autosampler and $5 \mu \mathrm{L}$ of sample was injected on the analytical column. The chromatographic separation was established using a SeQuant ZIC-cHILIC column (PEEK $100 \times 2.1 \mathrm{~mm}, 3.0 \mu \mathrm{m}$ particle size; Merck $\mathrm{KGaA}$, Darmstadt, Germany), which was kept at $15{ }^{\circ} \mathrm{C}$ using a column thermostat (560-CIL, Cleuzeau Info Labo, France). The flow rate was $0.2 \mathrm{~mL} / \mathrm{min}$. The mobile phases where composed of (A) $90 / 10$ acetonitrile $/ \mathrm{H}_{2} \mathrm{O}$ with $5 \mathrm{mM}$ ammonium acetate at $\mathrm{pH} 6.8$ and (B) 10/90 acetonitrile/ $\mathrm{H}_{2} \mathrm{O}$ with $5 \mathrm{mM}$ ammonium acetate at $\mathrm{pH} 6.8$, respectively. Metabolites were separated using a gradient composed of $100 \% \mathrm{~A}$ for $3 \mathrm{~min}$; ramping 3-20 $\mathrm{min}$ to $36 \% \mathrm{~A}$; ramping $28-28.5$ to $100 \% \mathrm{~A}$ and re-equilibrated from 28.5 to $36 \mathrm{~min}$ with $100 \% \mathrm{~A}$. The MS data was acquired at full scan range 50-800 amu at a scan rate of $10 \mathrm{scans} / \mathrm{s}$ in negative ionisation mode and the source temperature was kept at $400{ }^{\circ} \mathrm{C}$.

\subsection{Data analysis and statistics}

The metabolite peaks were integrated using MultiQuan Software v3.0 (SCIEX, Framingham, MA, USA). The responses of the targeted metabolites were corrected for the response of a selected internal standard. Statistical analysis was performed using GraphPad Prism 6 (Graphpad, La Jolla, CA, USA) and statistical programming language R (http:// www.r-project.org), with packages 'mixOmics' (Rohart et al. 2017) for the PLS-DA, and 'gplots' (https://cran.r-proje ct.org/package $=$ gplots) to visualize the heatmaps. Differences in individual metabolites were evaluated using a oneway analysis of variance (ANOVA) with a Tukey post-hoc correction for multiple comparisons. A $P$ value of $<0.05$ was considered significant.

\section{Results}

\subsection{ZIC-cHILIC-MS optimization for SCN and PVN}

The ZIC-cHILIC-MS method was partly adapted from a previous application of the ZIC-cHILIC column for serum metabolite profiling of colorectal tumor patients (Zhu et al. 2014) and modified for the measurement of anionic polar metabolites in SCN and PVN samples. For the sensitive measurement of the anionic polar metabolites in these samples, we optimized the extraction procedure for small sample sizes as described in Sect. 2. After metabolite extraction from the SCN and PVN, we were able to semi-quantitatively determine 35 anionic polar metabolites in these samples (Table S1 and Fig. S1). Polar metabolites were retained on the ZIC-cHILIC column and chromatographic separation was obtained (Fig. S1). For most of the metabolites, there was base-line separation and sensitivity was high enough to perform semi-quantitative analysis on the measured metabolites when using appropriate reconstructed ion chromatograms (Table S1). To determine ion suppression, we performed a post-column infusion experiment and did not observe any significant ion suppression (data not shown).

\subsection{Endogenous and induced electrical activity affects metabolites in SCN and PVN tissue}

To study the effect of time of day and of neuronal electrical activity in SCN and PVN tissue, we measured metabolites at two time-points, midday (ZT6) and midnight (ZT18), and in part of the brain slices we chemically manipulated the electrical activity. Intrinsic electrical activity in the SCN 
is high during the day, and low during the night, and the electrical activity in the PVN has been found to be in phase with the SCN (Tousson and Meissl 2004). Neuronal activity was blocked at midday with tetrodotoxin (TTX). TTX blocks $\mathrm{Na}^{2+}$-channels, preventing the initiation of action potentials, but does not affect membrane potential. Neuronal activity was induced at midnight by using increased extracellular $\mathrm{K}^{+}$ concentrations, which depolarizes the neuronal membrane, increasing the chance of the initiation of action potentials.

In order to understand the effect of the changes in metabolite levels due to tissue treatment, we applied a PLS-DA analysis to the ZIC-cHILIC-MS dataset. This resulted in a clear separation of the night High $\mathrm{K}^{+}$group and to a lesser degree of the control day group from the TTX and control night group (Fig. 2a). The TTX and control night group could not be separated by the PLS-DA, which is not unexpected, since the TTX manipulation was intended to simulate the night conditions for neurons in the SCN and PVN. The heatmap in Fig. $2 b$ represents the data for all the identified and measurable metabolites for all biological samples of this study, ordered on Variable Importance in Projection (VIP) score for the PLS-DA (Chong and Jun 2005). Interestingly, all the intermediates of the TCA cycle were among the metabolites with the highest VIP score.

\subsection{TCA cycle intermediates are upregulated after exposure to high extracellular $\mathrm{K}^{+}$}

Given the high VIP scores for the intermediates of the TCA cycle on the PLS-DA, we performed additional analysis on these metabolites (Fig. 3). From the metabolites directly involved in the TCA cycle, we could measure citrate, cisaconitate, succinate, fumarate and malate, which were all upregulated by exposure to high extracellular $\mathrm{K}^{+}$, compared to the other groups, both in the SCN and PVN (citrate, cisaconitate, succinate, malate $P<0.0001$, fumarate $P<0.01$ ). Malate was significantly higher in the control-day group, compared to the control-night group $(P<0.5)$. Thus, upregulating electrical activity by exposure to higher extracellular $\mathrm{K}^{+}$levels resulted in a strong upregulation of all measurable intermediates of the TCA cycle. In samples taken at midday compared to midnight, we could detect a significant difference for the TCA intermediate malate only.

\section{Discussion}

In this study we demonstrate the feasibility to measure metabolites in small tissue samples with ZIC-cHILIC-MS. We were able to semi-quantitatively measure 35 metabolites in the small tissue samples of the SCN and PVN (Table S1). Furthermore, we show that exposure of SCN and PVN tissue to high extracellular $\mathrm{K}^{+}$levels at night results in upregulation of many of the measured metabolites, and in particular of TCA cycle intermediates (Fig. 3; Figs. S2-S5).

Metabolites were measured in SCN and PVN tissue at midday (ZT6) and midnight (ZT18). In the SCN there is a strong circadian rhythm in neuronal activity, which peaks at midday and is lowest at midnight (Inouye and Kawamura 1979; Sato and Kawamura 1984). In vitro MUA recordings in the PVN have shown that its neuronal activity depends on AVP released from the SCN, but that the rhythm of electrical activity is in phase with that of the SCN (Tousson and Meissl 2004). To assess the impact of neuronal activity on metabolites, firing frequency was modulated in the SCN and PVN to simulate either a midday or midnight situation (Fig. 4). For this purpose, TTX was applied to silence the neurons of the SCN and PVN during the day, and elevated extracellular $\mathrm{K}^{+}$was applied to depolarize the neurons and increase firing frequency during the night. We found that the exposure to a higher $\mathrm{K}^{+}$concentration significantly upregulated all measured intermediates of the TCA-cycle in both the SCN and PVN. This suggests a higher energy demand of the neurons, as is previously described (Shetty et al. 2012). Consequently, we expected glycolysis to be upregulated in these samples, however, no significant differences in glycolysis intermediates was observed (Fig. S2). This indicates that different pathways from either fatty acids (fatty acid oxidation) or amino acids are used for energy metabolism under these conditions.

Only one of the measurable TCA cycle intermediates we found was significantly different between midday and midnight, and none were significantly different between midday and TTX treatment. This is surprising since cells are also more electrically active during the day than during the night or when silenced by with TTX (Shibata et al. 1984, 1982). However, the action potential frequency in SCN neurons is overall relatively low compared to other neurons, with a maximum of around $5 \mathrm{~Hz}$ during the day, and around $2.5 \mathrm{~Hz}$ during the night (Schaap et al. 1999). We do see a trend towards upregulation of the metabolites in the control-day group, compared to TTX treated and control-night samples (Fig. 2b). However, it is clear that high $\mathrm{K}^{+}$upregulates most of the metabolites we measured more severely than is the case for control-day vs TTX-day or control-night. An explanation could be that there is a greater increase in electrical activity as a consequence of the manipulation with high $\mathrm{K}^{+}$, compared to normal electrical activity at midday. This is perceivable, since high $\mathrm{K}^{+}$pulses have been used to mimic light pulses (Eskin 1972; Mirmiran et al. 1995; Schwartz 1991), and light pulses can elicit a response in firing frequency up to $25 \mathrm{~Hz}$ in SCN neurons (Irwin and Allen 2007; Meijer et al. 1998). An additional explanation could be that there is a homeostatic control of energy metabolites to anticipate the change in energy demand from night to day. In that case silencing the neurons with TTX might have a minimal effect 


\section{A $\mathrm{SCN}$}

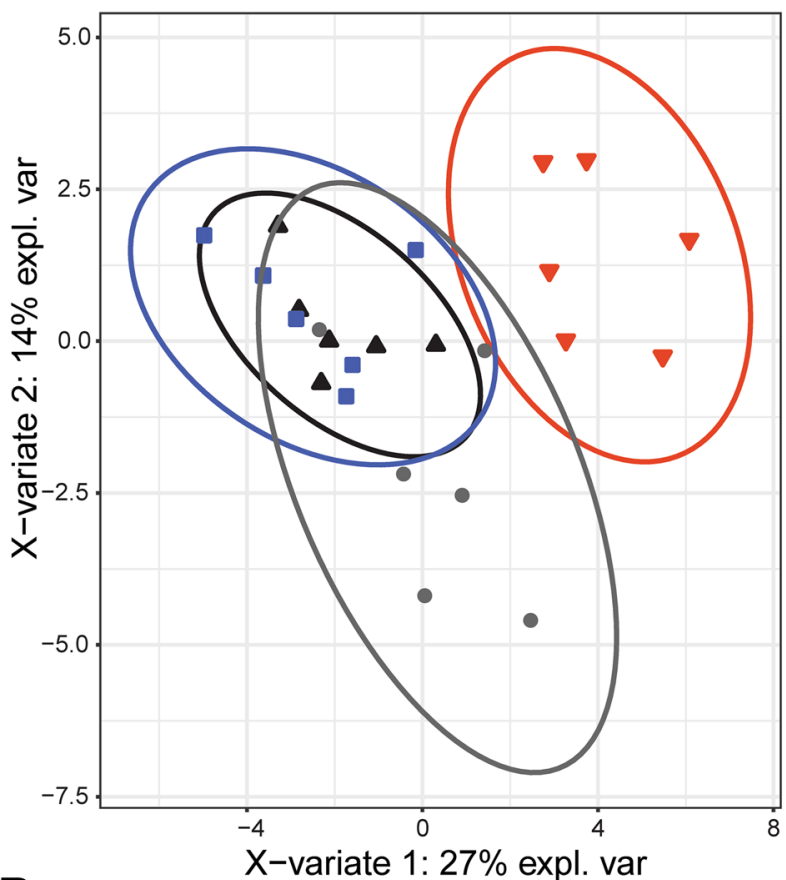

B SCN

control-day TTX-day control-night $\mathrm{HighK}^{+}$-night

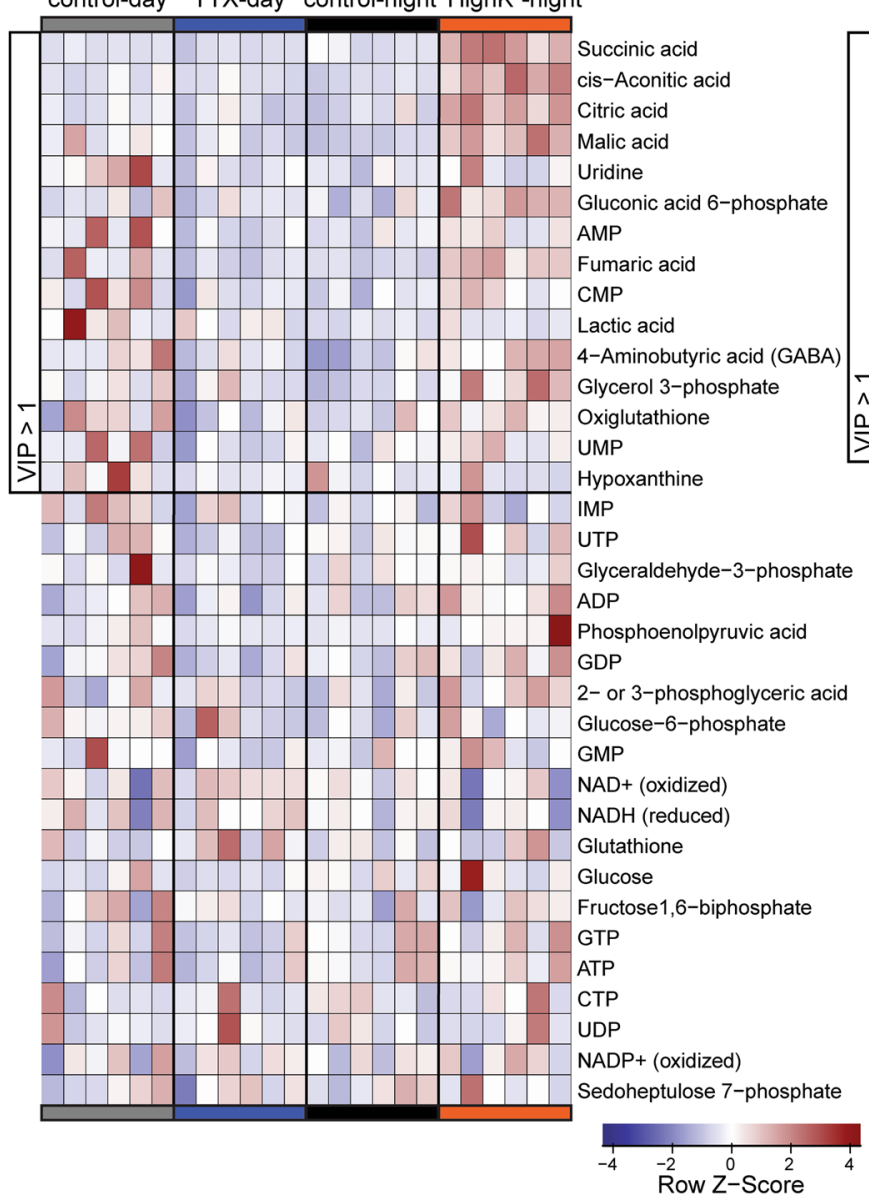

PVN

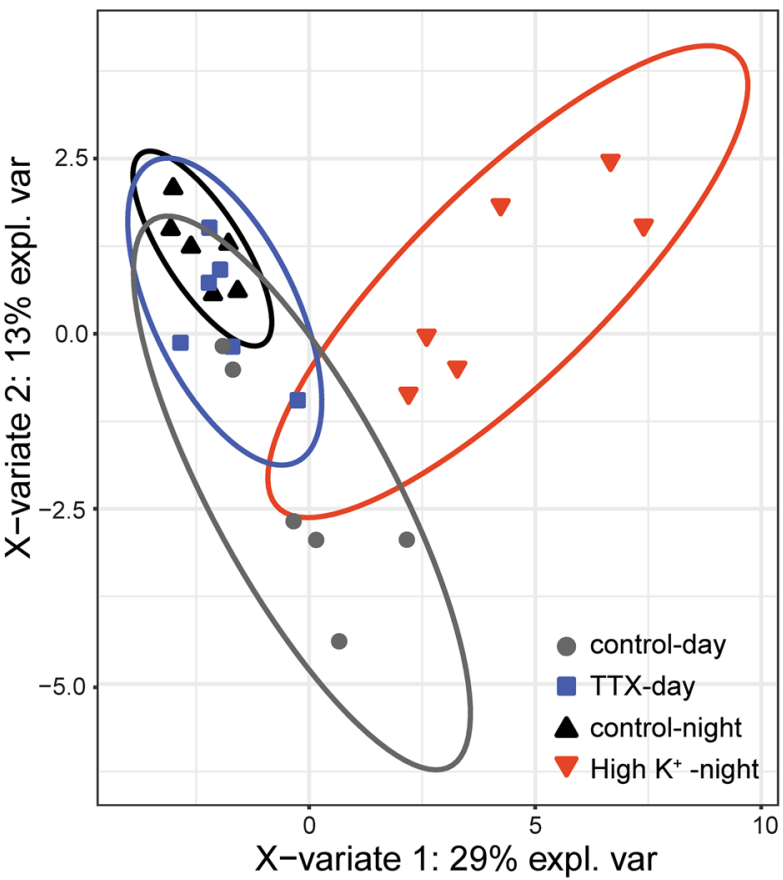

PVN

control-day TTX-day control-night $\mathrm{HighK}^{+}$-night

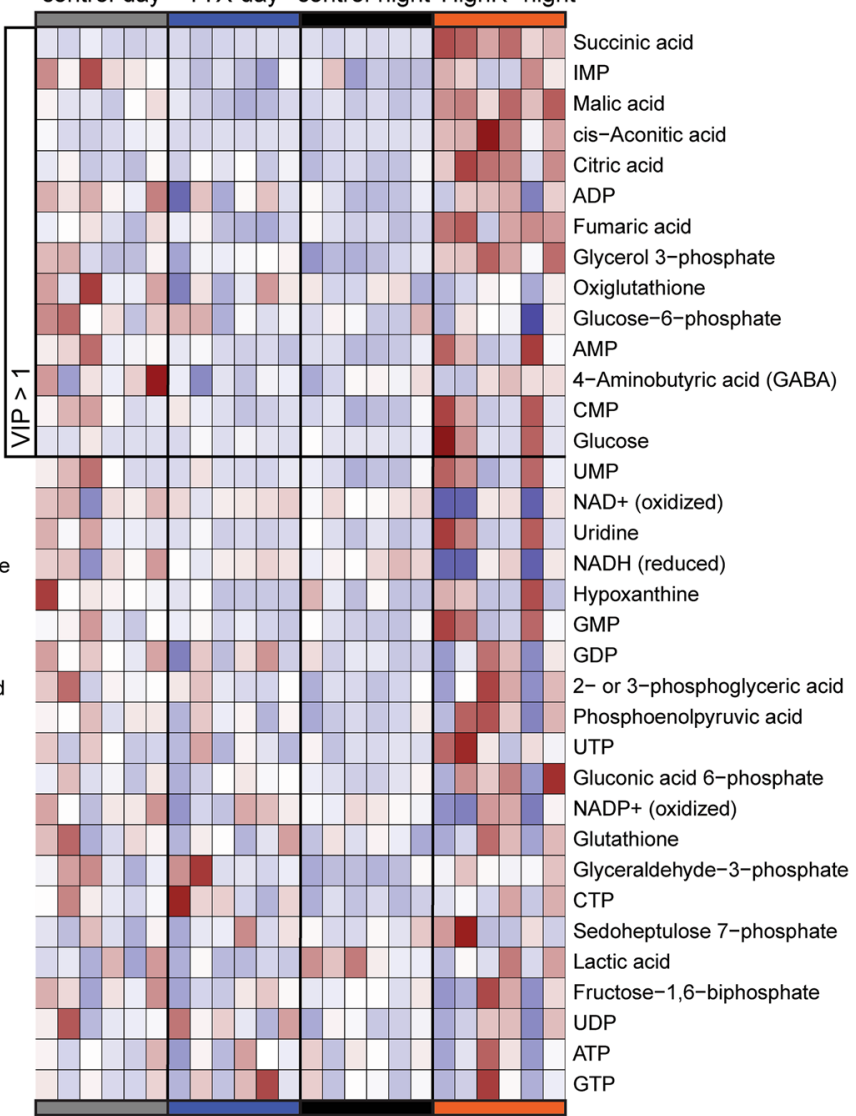


४Fig. 2 Global analysis of the complete data set. a An PLS-DA analysis separated the high $\mathrm{K}^{+}$-night group clearly, and to a lesser extend also separated the control-day group. The TTX-day and control-night groups largely overlap. Since silencing the neurons of the SCN with TTX at midday was intended to imitate the midnight state of the SCN this is in line with expectations. The results are similar for the SCN and PVN. $\mathbf{b}$ The contribution of the individual metabolites were calculated from the PLS-DA, giving a value of the VIP. The metabolites are ordered on their VIP score and their relative amount per individual sample shown in a heat plot. Among the metabolites with high VIP scores are all measurable metabolites of the TCA cycle, and several from the glycolysis pathway

on metabolite levels. Furthermore, individual neurons are naturally also inactive for a large proportion of the day; they are generally only active for about $5 \mathrm{~h}$ during daytime, and silent for the remaining $19 \mathrm{~h}$ (Schaap et al. 2003; VanderLeest et al. 2007).

The relatively strong response to the manipulation of electrical activity at midnight may be linked to the circadian fluctuation in the sensitivity to light pulses (Meijer et al. 1998). Light signals that reach the SCN from the eye through the RHT elicit electrical responses in SCN neurons. Experiments both in vivo-with light exposure-as well as in vitroby electrically stimulating the RHT-have shown that this only results in a phase shift in behaviour or in the electrical activity rhythm when received during the subjective night, not when the same signal is received during the subjective day (Shirakawa and Moore 1994). It could be that the strong response in metabolite levels to increased electrical activity at midnight is a means for the cells to support intracellular signalling pathways that generate light-induced phase shifts of the molecular clock.

The absence of detectible differences between metabolite levels at midday and midnight can have several reasons. First, if the peak and trough of the waveform is $6 \mathrm{~h}$ shifted from our sampling time points, there will be no differences detected. If the peak and trough are anywhere between 0 and $6 \mathrm{~h}$ from the sampling time points, this will result in smaller differences between the two measurements. Furthermore, the SCN and PVN are heterogeneous population of cells-including different types of neurons, as well as astrocytes-with diverse phases in their individual circadian rhythms (Abrahamson and Moore 2001; Brancaccio et al. 2017; Enoki et al. 2017; Tasker and Dudek 1991). Therefore, metabolites that are rhythmic in single cells, can be arrhythmic at the tissue level. Lastly, the one-hour incubation in ACSF may influence metabolite level in some unanticipated way. However, this is unlikely, since this incubation time does not affect rhythms in other physiological parameters of (SCN) neurons, like membrane potential, firing rate, intracellular $\mathrm{Ca}^{2+}$ levels or synaptic activity (Nakamura et al. 2012).

In this study, we looked at static basic metabolism. To gain more information on the involvement of energy balance, metabolic flexibility and substrate specificity of the SCN and PVN tissue, it could be interesting to follow metabolic fluxes by using isotopically stable labelled metabolites through the neuronal networks of the SCN and PVN under similar conditions as was described in this study. Using this technique, it would be possible to determine the real flux for the TCA cycle and which energy metabolites, glucose, fatty acids or amino acids, are involved for the energy utilization in neurons under high $\mathrm{K}^{+}$stimulation.

We show here that it is feasible to measure metabolites in small samples of brain tissue with a broadly applicable method, making it usable to tackle diverse biological questions. Using this method, we show that time of day, and manipulation of electrical activity affects metabolite levels in SCN and PVN tissue. Increasing electrical activity at midnight strongly upregulates metabolites of the TCA cycle. Our metabolomic study in the central circadian clock offers a unique view into the cellular biochemistry. Metabolites are not only a consequence of physiological states, they can actively influence cellular signalling pathways, including those involved in clock functioning. By combining this analytical tool with endogenous and exogenous modulation of neuronal activity, we were able to show the differential effect of these two sources of excitability on metabolites involved in cellular energy balance and signalling in the SCN. 
Fig. 3 Metabolites of the TCA cycle are mainly affected by incubation in high $\mathrm{K}^{+}$. a From the main metabolites of the TCA cycle, five were reliably measurable with the ZICcHILIC-MS method (boxed). b In both the SCN and PVN, all these metabolites were significantly upregulated by incubation in high $\mathrm{K}^{+}$-medium at midnight. In the SCN, there was higher level of malate in the control day group, compared to control night. $* P<0.05$, $* * P<0.01, * * * P<0.001$

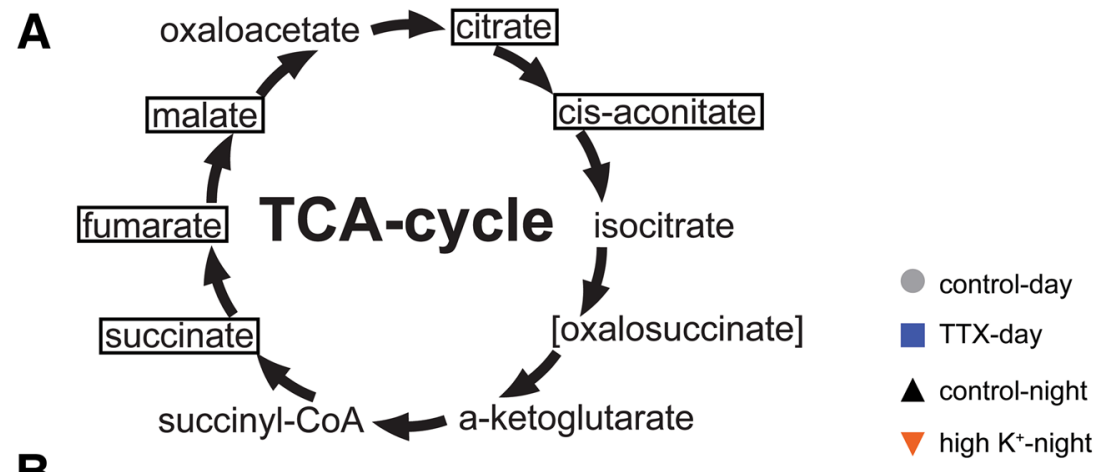

B

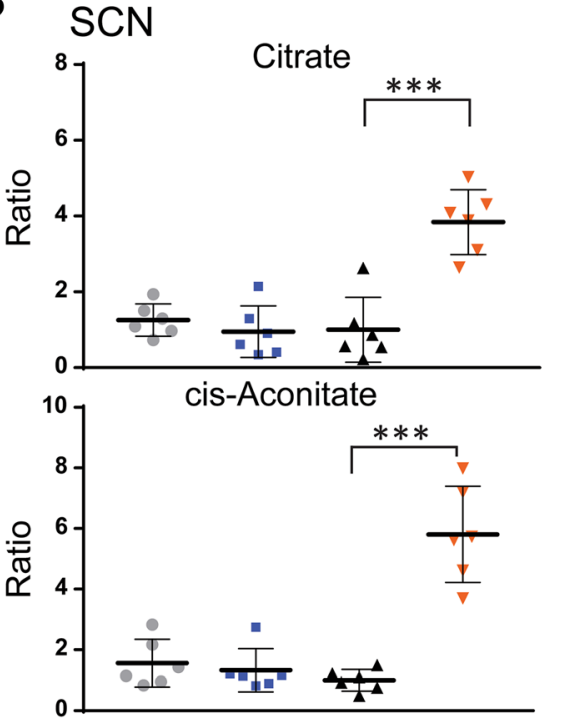

PVN Citrate
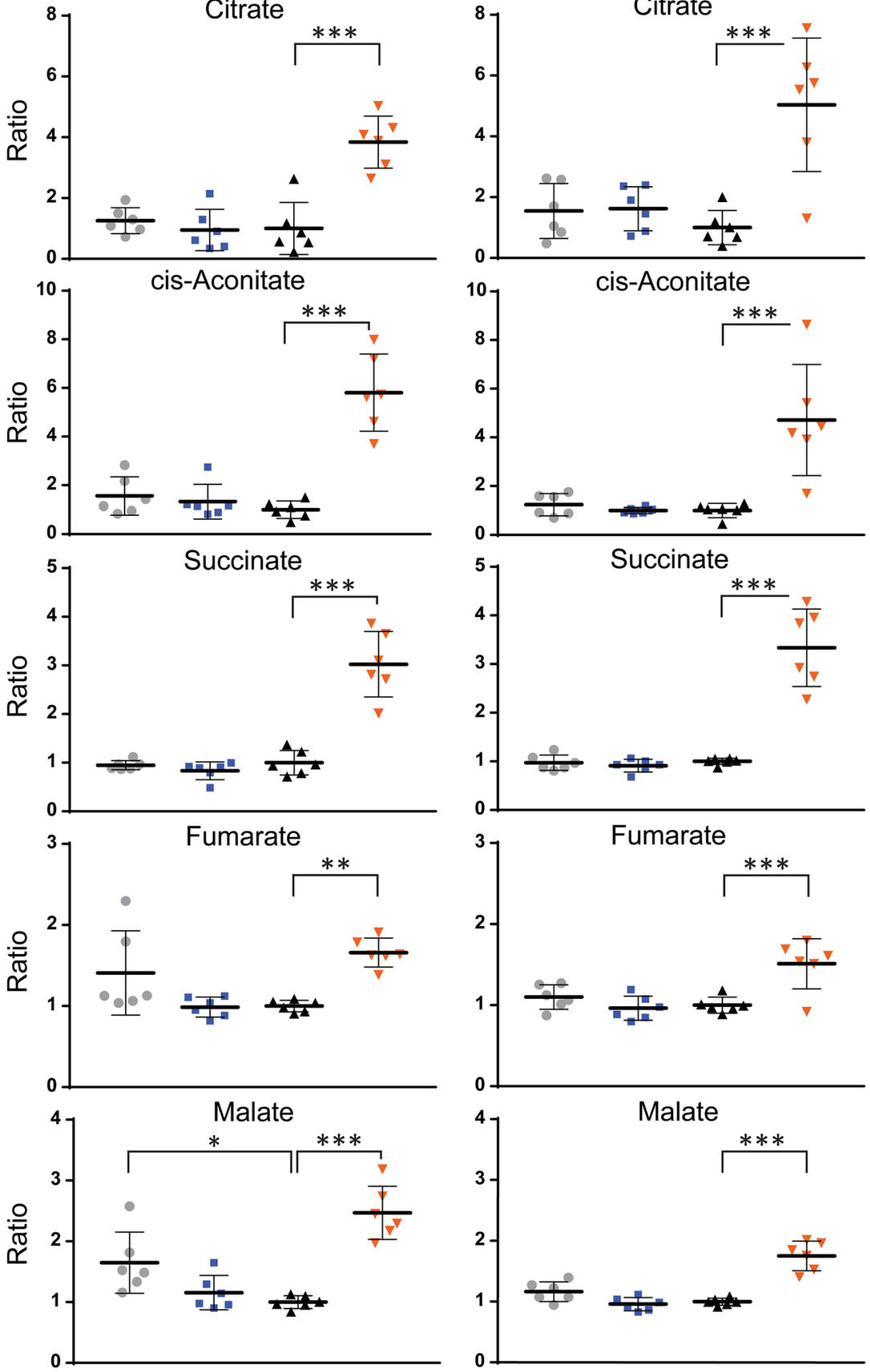


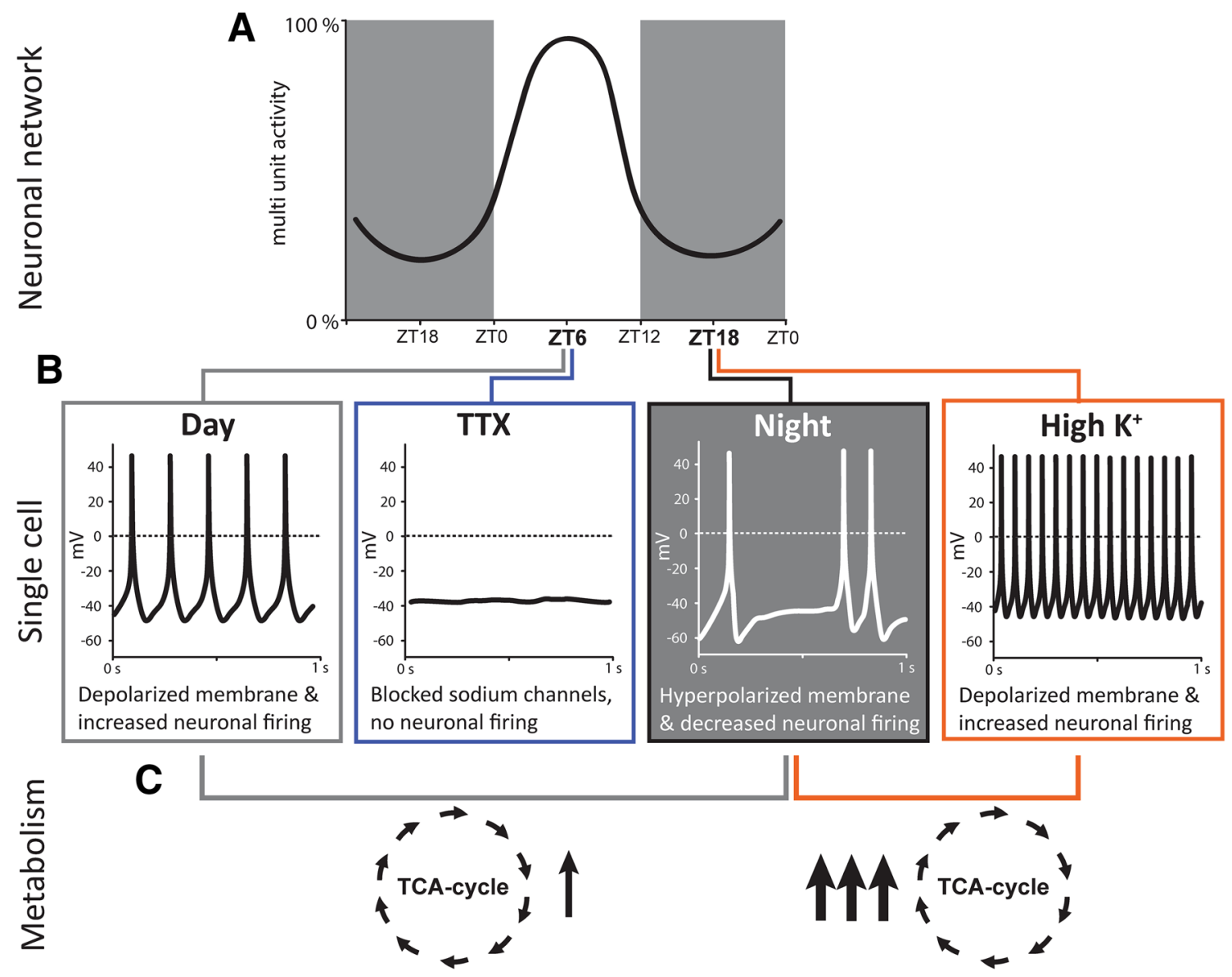

Fig. 4 Schematic overview of electrical activity in the SCN at the time of the experiments and its effect on metabolism. a On the network-level, the multi-unit activity of the SCN reaches its peak during the light period, between ZT0 (start light period) and ZT12 (end light period). b At the time of sampling, at ZT6 (day), most individual neurons are electrically active, and fire at a frequency of around $8 \mathrm{~Hz}$. At this time point, neurons were completely silenced by applying tetrodotoxin, which blocks sodium channels. At the second sam-

Acknowledgements We like to thank Fasia Guled and Mayke Tersteeg for their technical support. This study was supported by funding from the Netherlands Foundation of Technology (STW; ONTIME 12191).

Author contributions $\mathrm{MRB}, \mathrm{MvW}, \mathrm{ACH}, \mathrm{TH}, \mathrm{SM}$ conceived and designed research. MRB, MvW, MCG and SM conducted experiments. $\mathrm{MRB}, \mathrm{MvW}$ and JHR analyzed data. MRB and MvW wrote the manuscript. All authors read and approved the manuscript.

Data availability statement The datasets generated during and/or analysed during the current study are available from the corresponding author on reasonable request.

\section{Compliance with ethical standards}

Conflict of interest The authors declare no conflict of interest.

Human and animal rights All applicable international, national, and/or institutional guidelines for the care and use of animals were followed. pling time point, ZT18 (night), most neurons are electrically silent, or fire at a low frequency $(\sim 2.5 \mathrm{~Hz})$. By increasing the extracellular $\mathrm{K}^{+}$, the membrane potential was depolarized, thereby increasing neuronal firing. c Exposure to high $\mathrm{K}^{+}$at midnight severely upregulated all metabolites of the TCA cycle. The difference between midday and midnight was smaller, with one of the five measurable metabolites upregulated

Open Access This article is distributed under the terms of the Creative Commons Attribution 4.0 International License (http://creativeco mmons.org/licenses/by/4.0/), which permits unrestricted use, distribution, and reproduction in any medium, provided you give appropriate credit to the original author(s) and the source, provide a link to the Creative Commons license, and indicate if changes were made.

\section{References}

Abrahamson, E. E., \& Moore, R. Y. (2001). Suprachiasmatic nucleus in the mouse: Retinal innervation, intrinsic organization and efferent projections. Brain Research, 916, 172-191.

Allen, C. N., Nitabach, M. N., \& Colwell, C. S. (2017) Membrane currents, gene expression, and circadian clocks. Cold Spring Harbor Perspectives in Biology 9.

Brancaccio, M., Patton, A. P., Chesham, J. E., Maywood, E. S., \& Hastings, M. H. (2017). Astrocytes control circadian timekeeping in 
the suprachiasmatic nucleus via glutamatergic signaling. Neuron, 93, 1420-1435.

Chong, I.-G., \& Jun, C.-H. (2005). Performance of some variable selection methods when multicollinearity is present. Chemometrics and Intelligent Laboratory Systems, 78, 103-112.

Colwell, C. S. (2011). Linking neural activity and molecular oscillations in the SCN. Nature Reviews, 12, 553-569.

Coomans, C. P., van den Berg, S. A. A., Lucassen, E. A., Houben, T., Pronk, A. C. M., van der Spek, R. D., Kalsbeek, A., Biermasz, N. R., Willems van Dijk, K., Romijn, J. A., \& Meijer, J. H. (2013). The suprachiasmatic nucleus controls circadian energy metabolism and hepatic insulin sensitivity. Diabetes, 62, 1102-1108.

Davies, S. K., Ang, J. E., Revell, V. L., Holmes, B., Mann, A., Robertson, F. P., Cui, N., Middleton, B., Ackermann, K., Kayser, M., Thumser, A. E., Raynaud, F. I., \& Skene, D. J. (2014). Effect of sleep deprivation on the human metabolome. Proceedings of the National Academy of Sciences, 111, 10761-10766.

Eckel-Mahan, K. L., Patel, V. R., Mohney, R. P., Vignola, K. S., Baldi, P., \& Sassone-Corsi, P. (2012). Coordination of the transcriptome and metabolome by the circadian clock. Proceedings of the National Academy of Sciences, 109, 5541-5546.

Enoki, R., Oda, Y., Mieda, M., Ono, D., Honma, S., \& Honma, K. I. (2017). Synchronous circadian voltage rhythms with asynchronous calcium rhythms in the suprachiasmatic nucleus. Proceedings of the National Academy of Sciences of the United States of America, 114, E2476-E2485.

Eskin, A. (1972). Phase shifting a circadian rhythm in the eye of Aplysia by high potassium pulses. Journal of Comparative Physiology, 80, 353-376.

Gillette, M. U., \& Reppert, S. M. (1987). The hypothalamic suprachiasmatic nuclei: Circadian patterns of vasopressin secretion and neuronal activity in vitro. Brain Research Bulletin, 19, 135-139.

Ibuka, N., \& Kawamura, H. (1975). Loss of circadian rhythm in sleep-wakefulness cycle in the rat by suprachiasmatic nucleus lesions. Brain Research, 96, 76-81.

Ikeda, M., Sugiyama, T., Wallace, C. S., Gompf, H. S., Yoshioka, T., Miyawaki, A., \& Allen, C. N. (2003). Circadian dynamics of cytosolic and nuclear $\mathrm{Ca}^{2+}$ in single suprachiasmatic nucleus neurons. Neuron, 38, 253-263.

Inouye, S. T., \& Kawamura, H. (1979). Persistence of circadian rhythmicity in a mammalian hypothalamic "island" containing the suprachiasmatic nucleus. Proceedings of the National Academy of Sciences, 76, 5962-5966.

Irwin, R. P., \& Allen, C. N. (2007). Calcium response to retinohypothalamic tract synaptic transmission in suprachiasmatic nucleus neurons. Journal of Neuroscience, 27, 11748-11757.

Ju, Y.-E. S., Lucey, B. P., \& Holtzman, D. M. (2013). Sleep and Alzheimer disease pathology: A bidirectional relationship. Nature Reviews Neurology, 10,115-119.

Kalsbeek, A., La Fleur, S., Van Heijningen, C., \& Buijs, R. M. (2004). Suprachiasmatic GABAergic inputs to the paraventricular nucleus control plasma glucose concentrations in the rat via sympathetic innervation of the liver. Journal of Neuroscience, 24, 7604-7613.

Kalsbeek, A., Scheer, F. A., Perreau-Lenz, S., La Fleur, S. E., Yi, C. X., Fliers, E., \& Buijs, R. M. (2011). Circadian disruption and SCN control of energy metabolism. FEBS Letters, 585, 1412-1426.

Kondratova, A. A., \& Kondratov, R. V. (2012). The circadian clock and pathology of the ageing brain. Nature Reviews, 13, 325-335.

Kubota, A., Inouye, S.-I. T., \& Kawamura, H. (1981). Reversal of multiunit activity within and outside the suprachiasmatic nucleus in the rat. Neuroscience Letters, 27, 303-308.

Lapainis, T., Rubakhin, S. S., \& Sweedler, J. V. (2009). Capillary electrophoresis with electrospray ionization mass spectrometric detection for single-cell metabolomics. Analytical Chemistry, 81, $5858-5864$.
Lee, J. E., Atkins, N. Jr., Hatcher, N. G., Zamdborg, L., Gillette, M. U., Sweedler, J. V., \& Kelleher, N. L. (2010). Endogenous peptide discovery of the rat circadian clock: A focused study of the suprachiasmatic nucleus by ultrahigh performance tandem mass spectrometry. Molecular and Cellular Proteomics, 9, 285-297.

Meijer, J. H., Watanabe, K., Schaap, J., Albus, H., \& Detari, L. (1998). Light responsiveness of the suprachiasmatic nucleus: Long-term multiunit and single-unit recordings in freely moving rats. Journal of Neuroscience, 18, 9078-9087.

Michel, S., Marek, R., Vanderleest, H. T., Vansteensel, M. J., Schwartz, W. J., Colwell, C. S., \& Meijer, J. H. (2013). Mechanism of bilateral communication in the suprachiasmatic nucleus. European Journal of Neuroscience, 37, 964-971.

Minami, Y., Kasukawa, T., Kakazu, Y., Iigo, M., Sugimoto, M., Ikeda, S., Yasui, A., van der Horst, G. T. J., Soga, T., \& Ueda, H. R. (2009) Measurement of internal body time by blood metabolomics. Proceedings of the National Academy of Sciences, 106, 9890-9895.

Mirmiran, M., Koster-Van Hoffen, G. C., \& Bos, N. P. A. (1995). Circadian rhythm generation in the cultured suprachiasmatic nucleus. Brain Research Bulletin, 38, 275-283.

Moore, R. Y. (2007). Suprachiasmatic nucleus in sleep-wake regulation. Sleep Medicine, 8(Suppl 3), 27-33.

Moore, R. Y., \& Eichler, V. B. (1972). Loss of a circadian adrenal corticosterone rhythm following suprachiasmatic lesions in the rat. Brain Research, 42, 201-206.

Musiek, E. S., Xiong, D. D., \& Holtzman, D. M. (2015). Sleep, circadian rhythms, and the pathogenesis of Alzheimer disease. Experimental and Molecular Medicine, 47, e148.

Nagai, K., Nishio, T., Nakagawa, H., Nakamura, S., \& Fukuda, Y. (1978). Effect of bilateral lesions of the suprachiasmatic nuclei on the circadian rhythm of food-intake. Brain Research, 142, 384-389.

Nakamura, T. J., Michel, S., Block, G. D., \& Colwell, C. S. (2012). Neural circuits underlying circadian oscillations in mammals: Clocks in a dish. In K. Ballanyi (Ed.), Isolated central nervous system circuits (pp. 183-210). Totowa: Humana Press.

Nemes, P., Knolhoff, A. M., Rubakhin, S. S., \& Sweedler, J. V. (2011). Metabolic differentiation of neuronal phenotypes by single-cell capillary electrophoresis-electrospray ionization-mass spectrometry. Analytical Chemistry, 83, 6810-6817.

Nishino, H., Kiyomi, K., \& Brooks, C. M. (1976). The role of suprachiasmatic nuclei of the hypothalamus in the production of circadian rhythm. Brain Research, 112, 45-59.

O’Neill, J. S., Maywood, E. S., Chesham, J. E., Takahashi, J. S., \& Hastings, M. H. (2008). cAMP-dependent signaling as a core component of the mammalian circadian pacemaker. Science, 320 , 949-953.

Qi, M., Philip, M. C., Yang, N., \& Sweedler, J. V. (2018). Single cell neurometabolomics. ACS Chemical Neuroscience, 9, 40-50.

Rey, G., Valekunja, U. K., Feeney, K. A., Wulund, L., Milev, N. B., Stangherlin, A., Ansel-Bollepalli, L., Velagapudi, V., O’Neill, J. S., \& Reddy, A. B. (2016). The pentose phosphate pathway regulates the circadian clock. Cell Metab, 24, 462-473.

Rohart, F., Gautier, B., Singh, A., \& Lê Cao, K.-A. (2017). mixOmics: An R package for 'omics feature selection and multiple data integration. PLoS Computational Biology, 13, e1005752.

Rudic, R. D., McNamara, P., Curtis, A. M., Boston, R. C., Panda, S., Hogenesch, J. B., \& Fitzgerald, G. A. (2004). BMAL1 and CLOCK, two essential components of the circadian clock, are involved in glucose homeostasis. PLoS Biology, 2, e377.

Sangoram, A. M., Saez, L., Antoch, M. P., Gekakis, N., Staknis, D., Whiteley, A., Fruechte, E. M., Vitaterna, M. H., Shimomura, K., King, D. P., Young, M. W., Weitz, C. J., \& Takahashi, J. S. (1998). Mammalian circadian autoregulatory loop: A timeless ortholog 
and mPer1 interact and negatively regulate CLOCK-BMAL1induced transcription. Neuron, 21, 1101-1113.

Santoso, P., Nakata, M., Ueta, Y., \& Yada, T. (2017). Suprachiasmatic vasopressin to paraventricular oxytocin neurocircuit in the hypothalamus relays light reception to inhibition of feeding behavior. American Journal of Physiology-Endocrinology and Metabolism. https://doi.org/10.1152/ajpendo.00338.2016.

Sato, T., \& Kawamura, H. (1984). Circadian rhythms in multiple unit activity inside and outside the suprachiasmatic nucleus in the diurnal chipmunk (Eutamias sibiricus). Neuroscience Research, $1,45-52$.

Schaap, J., Albus, H., vanderLeest, H. T., Eilers, P. H. C., Détári, L., \& Meijer, J. H. (2003) Heterogeneity of rhythmic suprachiasmatic nucleus neurons: Implications for circadian waveform and photoperiodic encoding. Proceedings of the National Academy of Sciences, 100, 15994-15999.

Schaap, J., Bos, N. P., de Jeu, M. T., Geurtsen, A. M., Meijer, J. H., \& Pennartz, C. M. (1999). Neurons of the rat suprachiasmatic nucleus show a circadian rhythm in membrane properties that is lost during prolonged whole-cell recording. Brain Research, $815,154-166$.

Schwartz, W. J. (1991). Further evaluation of the tetrodotoxin-resistant circadian pacemaker in the suprachiasmatic nuclei. Journal of Biological Rhythms, 6, 149-158.

Shetty, P. K., Galeffi, F., \& Turner, D. A. (2012) Cellular links between neuronal activity and energy homeostasis. Frontiers in Pharmacology 3 .

Shibata, S., Oomura, Y., Hattori, K., \& Kita, H. (1984). Responses of suprachiasmatic nucleus neurons to optic nerve stimulation in rat hypothalamic slice preparation. Brain Research, 302, 83-89.

Shibata, S., Oomura, Y., Kita, H., \& Hattori, K. (1982). Circadian rhythmic changes of neuronal activity in the suprachiasmatic nucleus of the rat hypothalamic slice. Brain Research, 247, $154-158$.
Shirakawa, T., \& Moore, R. Y. (1994). Glutamate shifts the phase of the circadian neuronal firing rhythm in the rat suprachiasmatic nucleus in vitro. Neuroscience Letters, 178, 47-50.

Stephan, F. K., \& Zucker, I. (1972) Circadian rhythms in drinking behavior and locomotor activity of rats are eliminated by hypothalamic lesions. Proceedings of the National Academy of Sciences, 69, 1583-1586.

Tasker, J. G., \& Dudek, F. E. (1991). Electrophysiological properties of neurones in the region of the paraventricular nucleus in slices of rat hypothalamus. The Journal of Physiology, 434, 271-293.

Tousson, E., \& Meissl, H. (2004). Suprachiasmatic nuclei grafts restore the circadian rhythm in the paraventricular nucleus of the hypothalamus. Journal of Neuroscience, 24, 2983-2988.

Turek, F. W., Joshu, C., Kohsaka, A., Lin, E., Ivanova, G., McDearmon, E., Laposky, A., Losee-Olson, S., Easton, A., Jensen, D. R., Eckel, R. H., Takahashi, J. S., \& Bass, J. (2005). Obesity and metabolic syndrome in circadian Clock mutant mice. Science, 308, 1043-1045.

VanderLeest, H. T., Houben, T., Michel, S., Deboer, T., Albus, H., Vansteensel, M. J., Block, G. D., \& Meijer, J. H. (2007). Seasonal encoding by the circadian pacemaker of the SCN. Current Biology, 17, 468-473.

Wang, T. A., Yu, Y. V., Govindaiah, G., Ye, X., Artinian, L., Coleman, T. P., Sweedler, J. V., Cox, C. L., \& Gillette, M. U. (2012). Circadian rhythm of redox state regulates excitability in suprachiasmatic nucleus neurons. Science, 337, 839-842.

Wulff, K., Gatti, S., Wettstein, J. G., \& Foster, R. G. (2010). Sleep and circadian rhythm disruption in psychiatric and neurodegenerative disease. Nature Reviews, 11, 589-599.

Zhu, J., Djukovic, D., Deng, L., Gu, H., Himmati, F., Chiorean, E. G., \& Raftery, D. (2014). Colorectal cancer detection using targeted serum metabolic profiling. Journal of Proteome Research, 13, $4120-4130$. 